\title{
SELECTIVIDAD DE MIGRANTES A UNA METRÓPOLI EN UN PAÍS EN DESARROLLO: ESTUDIO DE UN CASO MEXICANO
}

\author{
HaRley L. Browning y Waltraur FeINDT \\ Universidad de Texas
}

UNO DE LOS PROBLEMAS constantes en el estudio de la migración al evajuar las diferencias entre nativos y migrantes es la selectividad de estos últimos. $i$ Los migrantes son selectivos positiva o negativamente, o no muestran diferencias al compararse con aquellos de las comunidades de origen? La dificultad surge del hecho de que casi todos los estudios sobre migrantes están limitados a una localidad, la de destino. En virtud de que los migrantes provienen de muchas comunidades, es obviamente muy difícil tratar de obtener información comparable para cada una. En consecuencia, el grado y la clase de selectividad del migrante son casi siempre desconocidos, aun cuando estas características son de indudable pertinencia en la interpretación de problemas tales como el éxito de los migrantes al competir con los nativos por alcanzar posiciones ocupacionales.

Dada esta situación, la mayor parte de las discusiones sobre selectividad de los migrantes ha sido de carácter especulativo e inferencial. Por fortuna, cuando menos en Estados Unidos, el estudio reciente, en gran escala, de Blau y Duncan brinda la mejor evidencia que jamás haya estado disponible sobre esta cuestión. Con base en los resultados de una muestra nacional representativa de más de 20000 hombres estadounidenses entre 20 y 64 años de edad, los autores mencionados llegaron a las siguientes conclusiones:

Ya sea que se examine la migración entre regiones o entre comunidades, o que se considere desde el nacimiento o desde la adolescencia, o que las comparaciones entre migrantes y no migrantes se hagan sobre agrupaciones étnicas y de natividad o sin emplear este control, o que la educación y el primer empleo se hayan mantenido constantes, o que los migrantes se comparen con los nativos del lugar de origen o de destino, los migrantes tienden a lograr niveles ocupacionales más altos y a experimentar movilidad más ascendente que la de los no migrantes, salvo unas cuantas excepciones. $x$

Estos hallazgos dan fuerte respaldo a la afirmación de que los migrantes son selectivos positivamente, es decir, que no son representativos de la comunidad de donde provienen. Pero el estudio de Blau

1 P. M. Blau y D. D. Duncan, The American Occupational Structure, Nueva York, John Wiley \& Sons, 1967, p. 272. 
y Duncan se refiere a un país altamente industrializado y surge la cuestión de si en los países en otras etapas de desarrollo se observa el mismo patrón. Específicamente, puede sostenerse que en los Estados Unidos las diferencias socioeconómicas entre regiones y entre áreas urbanas y rurales se han reducido sustancialmente; en contraste con muchos países en desarrollo en los que las diferencias de niveles socioeconómicos entre las áreas rurales, eminentemente campesinas, y las ciudades de crecimiento rápido y en proceso de industrialización son muy pronunciadas. Dadas estas diferencias, la selectividad de los migrantes puede ser también distinta.

Este estudio se enfoca al problema anterior a través de una investigación de la selectividad de migrantes en una metrópoli industrial de rápido crecimiento (Monterrey) en un país en desarrollo (México). Nuestro interés radica en el grado de selectividad de los que emigran a Monterrey cuando se comparan éstos con la población de sus comunidades de origen. (No se considera aquí qué tan bien se hallan los migrantes una vez llegados a Monterrey, comparados con los nativos. Esto se trata en otro trabajo de los autores.) Se plantean dos cuestiones principales. Primera: ¿hay selectividad en los migrantes? Específicamente, ¿qué diferencias en grado de educación y en posición ocupacional existen entre aquéllos y las poblaciones comparables de donde provienen? Segundo: suponiendo que sí hay tal selectividad, ¿ha cambiado ésta en el tiempo? En otras palabras, ¿son los migrantes más recientes más o menos selectivos que los que llegaron anteriormente?

\section{INFORMACION Y PROCEDIMIENTOS}

La información para este estudio se tomó de una encuesta llevada a cabo durante el verano de 1965 en relación con 1640 hombres entre 21 y 60 años de edad, residentes en el área metropolitana de Monterrey, México. La muestra fue de diseño bietápico de conglomerado estratificado, ponderada en favor de los hombres de más edad (entre 41 y 60) y de los de ingresos más elevados.2 La información se obtuvo mediante preguntas específicas y a través de un largo esquema que contenía la historia vital completa del entrevistado en relación con variables tales como educación, empleo, y todos los cambios de residencia de seis meses o más de duración. ${ }^{3}$ Esta información permite dirigirnos a la segunda cuestión principal porque nos capacita para "ubicar" las características socioeconómicas de los migrantes en cual. quier momento de sus vidas.

En este artículo sólo nos referimos a los migrantes; la distinción entre éstos y los nativos se basa no en el lugar de nacimiento del entrevistado sino en su "comunidad de origen", es decir, el lugar donde vivió durante sus años formativos, definidos aquí como los vividos entre las edades de cinco y quince años. De nuestra muestra, $56 \%$

2 Véase Centro de Investigaciones Económicas, Facultad de Economía, Universidad de Nuevo León, Movilidad Social, Migración y Fecundidad en Monterrey Metropolitano. Monterrey, 1967. Cap. I.

3 Sobre el método de historia vital, véase Jorge Balán et al., "El uso de computadoras en el análisis de historias vitales", DEMOGRAFfa Y ECONOMfA, Vol. II, Núm. 3 , 1968, pp. $428-442$. 
(907 personas) habían nacido fuera de Monterrey o no era ésta su comunidad de origen. El $14 \%$ (228 personas) nacieron fuera de Monterrey pero emigraron a esta ciudad durante su niñez y pasaron allí sus años formativos. A estos últimos no los consideramos migrantes sino "nativos por adopción". Hay, en la muestra, 165 migrantes que regresaron: aquellos que clejaron la ciudad durante seis meses o más pero que en 1965 ya habían regresado. Sin embargo, para el $81 \%$ de los migrantes (739), la primera y la última llegada son idénticas. Cuando digamos "llegada a Monterrey", nos referiremos a la primera.

El período de arribo a Monterrey fue establecido mediante tres cohortes de llegada: antes de 1940, entre 1941 y 1950 y entre 1951 y 1960. Deben mencionarse ciertos aspectos de estas cohortes. Primero, nuestra muestra está restringida a hombres entre 21 y 60 en la fecha de la encuesta, 1965; esto quiere decir que para la cohorte de llegada más temprana (antes de 1941) tenemos un grupo restringido por las edades, ya que ninguno de los hombres de esta cohorte pudo haber tenido más de 40 años cuando llegaron por primera vez a Monterrey. De la misma manera, los hombres de la segunda cohorte (1941-1950) no pudieron haber tenido más de 50 años. Afortunadamente, en Monterrey como en cualquier otra parte, la migración es relativamente poco frecuente en edades por encima de los 40, de manera que la exclusión de estos hombres de más edad representa sólo una pequeña proporción de la cohorte potencial.

Un problema más grave es el de la extinción de las cohortes y se debe a dos causas: la mortalidad y la emigración. La primera es significativa sólo si suponemos que hubo mortalidad diferencial muy acentuada entre las tres cohortes y hay pocas razones para pensar eso. Pero el problema de los que dejaron Monterrey es esencial en esta investigación porque se debe considerar la posibilidad de que los migrantes de nuestra muestra sean a su vez selectivos entre todos los migrantes que llegaron a Monterrey. Uno puede sostener que hay un sesgo simétrico en la comparación de las cohortes por fecha de llegada porque mientras más temprana sea ésta, más selectivo es el grupo, debido a la salida de los que tienen menos éxito. Sin embargo, esta limitación no es tan seria en la práctica como parece serlo en la teoría, más que nada porque hemos excluiclo del análisis la cohorte más inestable, la de aquellos que llegaron por primera vez entre 1961 y 1965. Todos los miembros de la cohorte de llegada más reciente tuvieron cuando menos cinco años de residencia en Monterrey.

En adición, tenemos una fuente independiente de información que nos lleva a creer que la mayor parte de la emigración sucede dentro de los primeros años después del arribo. En 1967 se llevó a cabo una encuesta en Cedral, una comunidad principalmente agrícola de aproximadamente 5000 habitantes situada al norte de San Luis Potosí, un área que contribuyó con el grupo más grande de migrantes a Monterrey. De los 380 hombres entre 15 y 64 años de edad que fueron entrevistados, casi la mitad había residido alguna vez en Monterrey, sólo

4 Véase Harley L. Browning y Waltraut Feindt, "Diferencias entre la población nativa y la migrante en Monterrev", DeMograffa y Economfa, Vol. II, Núm. 2 , 1968, pp. 183-204. 
para regresar a la comunidad de Cedral a los dos años de su llegada a Monterrey.

Otra razón para excluir las llegadas 1961-1965 es la circunstancia de que este grupo contiene un número significativo de hombres ocupacionalmente en tránsito. El personal militar, individuos transferidos debiclo a sus negocios, comisionados políticos, especialistas del gobierno, etc., son ejemplo de individuos que vienen a Monterrey sin la intención de establecerse allí permanentemente y la gran mayoría de ellos dejan la ciudad antes de cinco años de estancia.

Finalmente, en nuestra discusión del posible sesgo de emigración, debe mencionarse que tenemos pocas razones para pensar que sólo los migrantes que no han tenido éxito dejan Monterrey. Aunque no tenemos información sobre su número, algunos migrantes a Monterrey que han tenido éxito, tiempo después emigran a la ciudad de México o tal vez a Guadalajara. Esto de manera alguna equivale a decir que la emigración de personas que tuvieron éxito iguala a aquella de los que no lo tuvieron, pero sí sugiere que el proceso no es uniforme. Creemos que al suprimir la cohorte de llegada 1961-1965, el peligro de efectos distorsionantes debido a la emigración selectiva queda reducido sustancialmente si no es que eleminado del todo.

\section{ORIGEN GEOGRAFICO DE LOS MIGRANTES}

Monterrey es el centro urbano más importante en el nordeste de México, y los cuatro estaclos de esta área (Coahuila, Nuevo León, San Luis Potosí y Tamaulipas) abastecen la gran mayoría de emigrantes a Monterrey. De los cuatro estados mencionados, todos salvo San Luis Potosí están entre los ocho más sobresalientes, en término de desarrollo socioeconómico general, de los treinta y dos estados mexicanos. 5 Uno puede suponer, por lo tanto, que Monterrey extrae sus migrantes de áreas homogéneas y de niveles de bienestar relativamente altos, hablando en términos socioeconómicos. Pero esta afirmación es válida sólo al nivel estatal, porque en México hay heterogeneidad considerable dentro de los estados. Afortunadamente, se cuenta con unidades mejores de análisis, las zonas, formadas por municipios (equivalentes a condados en Estados Unidos) relativamente homogéneos. ${ }^{6}$ Las 111 zonas de México fueron ordenadas con información del censo de 1960 de acuerdo con tres indicadores de desarrollo económico: porciento de población urbana, porciento de fuerza de trabajo en actividades terciarias y secundarias e ingreso por trabajador. Dentro de esta ordenación, las zonas se agruparon en cinco categorías, la I la más alta y la V la más baja (ver cuadro 1). La categoría I está formada por zonas de carácter altamente urbano, generalmente metropolitano. En contraste, la categoría $V$ es fuertemente rural y muy atrasada en todos sus aspectos.

El cuadro 1 muestra que nuestro supuesto inicial no es válido: Monterrey atrae migrantes de todas las zonas socioeconómicas, no (v)

5 P. Lamartine Yates, El desarrollo regional de México. México, Banco de México, Depto. de Investigaciones Industriales, 1961.

B Claudio Stern, "Un análisis regional de México", Demografía Y Economía, Vol. I, Núm. 1, 1967, pp. 92-117. 
sólo de las más desarrolladas. En efecto, al combinar las categorías I y II y I y V se observará que hay, aproximadamente, tantos migrantes de las categorías más avanzadas como de las más atrasadas (alrededor de $40 \%$ ), y que su contribución en el tiempo permanece estable. E1 principal cambio en el tiempo, sin embargo, se da en las categorías menos desarrolladas: la IV declinó en importancia mientras que la $\mathrm{V}$ aumentó.

\section{Cuadro 1}

DistribUCIÓN DE EMIGRANTES A MONTERREY AGRUPADOS POR ZONAS SOCIOECONOMICAS DE LAS COMUNIDADES DE ORIGEN Y. POR FECHAS DE PRIMER ARRIBO

(En porcientos)

\begin{tabular}{|c|c|c|c|}
\hline Zonas socioosonóntas & Antes de 1941 & $1941-1950$ & $1951-1960$ \\
\hline Categoria I (Suparior). & 22 & 20 & 29 \\
\hline Categoria II & 18 & 20 & 23 \\
\hline Categoria III & 18 & 27 & 20 \\
\hline Categoria IV & 36 & 34 & 27 \\
\hline Categoria V(Inferior) & 6 & 20 & 12 \\
\hline $\begin{array}{l}\text { Total } \\
\text { N }\end{array}$ & $\begin{array}{c}100 \\
(200)\end{array}$ & $\begin{array}{c}101 \\
(250)\end{array}$ & $\begin{array}{c}102 \\
(304)\end{array}$ \\
\hline
\end{tabular}

* Todas las zonas están representadas. Una lista de las áreas de mayor contribución en cada categoría aparece en el cuadro 2.

\section{SELECTIVIDAd OCUPACTONAL Y EDUCATIVA}

Aunque es útil saber que los migrantes a Monterrey provienen, más - menos al parejo, de áreas geográficas de niveles socioeconómicos bajos y altos, esta información por sí misma no da respuesta a la pregunta principal pianteada en este estudio: ¿Son los que emigran a Monterrey selectivos respecto de las poblaciones de donde provienen? Como se indicó, la única manera del todo satisfactoria de atacar este problema sería efectuar encuestas comparables a la de Monterrey en todas las comunidades de origen de los migrantes a esa ciudad. Esto, desde luego, no fue posible; sin embargo, puede ganarse cierta comparación, aunque imperfecta, al confrontar los resultados de la encuesta de Monterrey con los del censo decenal de México referente a educación y ocupación. Tenemos las zonas (agregados de municipios) de origen de los individuos así como la fecha de su llegada y en lo referente a educación, podemos estar seguros de que casi todos los individuos completaron la primaria (los primeros seis años de escuela) en sus comunidades de origen y no en alguna otra, ya que hemos definido éstas como aquellas en donde el entrevistado residió durante la mayor 
parte del período comprendido entre los cinco y los quince años de edad. El status ocupacional es mucho más ambiguo porque el indicador usado es el porciento de hombres empleados en actividades no agrícolas durante el año anterior al arribo a Monterrey, y esto quiere decir que un número desconocido de hombres vivía en comunidades distintas de la de origen el año anterior a la llegada.

Dada la información anterior, nuestro procedimiento será comparar las características ocupacionales y educacionales de los migrantes a Monterrey con la información comparable referente a los individuos de las zonas socioeconómicas, correspondiente a los censos de 1940 y 1960. (Los resultados del censo de 1950 no son comparables.) Los intervalos de edad de los dos conjuntos de datos no son idénticos; para Monterrey es 21-60, mientras que para los dos períodos censales todos son hombres de más de 15 años. No obstante, debido a los niveles de educación generalmente bajos y la edad temprana de ingreso a la actividad en México, así como a la relativa escasez de hombres de más de 60 años, la discrepancia no debe ser restricción importante sobre la comparabilidad.

El cuadro 2 contiene información sobre niveles ocupacionales. Escogimos primaria completa (seis años o más de escuela) por varias razones. Por una parte, la primaria es la primera etapa completa del proceso de educación en México y la primaria completa confiere un certificado que se exige cada vez más al solicitar empleo en México. En realidad, alcanzar este nivel aun significa poseer un grado educativo mucho mejor que el promedio ya que, en 1960, sólo cerca de un quinto de la población masculina mayor de 15 años había alcanzado tal nivel.

El cuadró 2 nos permite dirigirnos no sólo a las cuestiones principales de este estudio - la selectividad y sus cambios en el tiemposino también a las diferencias por categoría de zona socioeconómica. Empecemos con la pregunta, "¿son los migrantes a Monterrey selectivos de los individuos comparables de las comunidades de donde provienen?" Las cifras totales del cuadro 2 demuestran en forma concluyente que sí lo son sustancialmente y en sentido positivo, lo cual significa que los migrantes tienen niveles educativos mucho más elevados que los que se encuentran en sus comunidades de or̆igen. Pero este hallazgo general esconde las diferencias importantes que existen cuando se consideran las cinco categorías de zonas socioeconómicas. Un examen de las columnas 6 y 7 del cuadro 2 , que muestran las diferencias en puntos de porciento entre dos cohortes de llegada y sus años censales relevantes, indicará que para las diez comparaciones todos los signos son positivos (los migrantes tienen mejor educación que las personas de sus comunidades de origen) salvo una. En la categoría I, los que llegaron antes de 1941 tuvieron una menor proporción de personas con seis años o más de educación que la población masculína total de más de 15 años. En contraste, en la categoría IV, el $51 \%$ de los que llegaron antes de 1941 tuvieron seis o más años de escuela en comparación con sólo el $5 \%$ en los censos.

Antes de intentar dar explicación de estos hallazgos vayamos a la segunda pregunta, "¿se han vuelto más selectivos los migrantes?" Una vez más nos referimos a las columnas 6 y 7 del cuadro 2; para la 


\section{Cuadro $\dot{2}$}

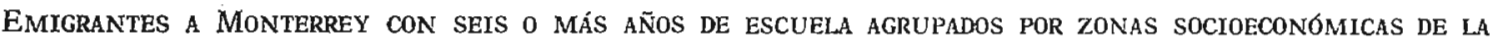
COMUNIDAD DE ORIGEN, POR FFCHA DE PRIMERA LLEGADA, COMPARADOS CON TODOS LOS INDIVIDUOS DE 15 Y MÁS AÑOS EN LAS MISMAS ZONAS TOMADAS DE LOS CENSOS DE 1940 Y 1960

(En porcientos)

\begin{tabular}{|c|c|c|c|c|c|c|c|}
\hline \multirow{2}{*}{ Zonus Socioeconómicas } & \multirow{2}{*}{ 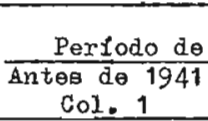 } & \multirow{2}{*}{$\begin{array}{c}\text { la primera } \\
1941-1950 \\
\text { Col. } 2\end{array}$} & \multirow{2}{*}{$\begin{array}{c}\text { Llegada } \\
1951-1960 \\
\text { Col. } 3\end{array}$} & \multirow{2}{*}{ 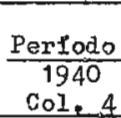 } & \multirow{2}{*}{$\frac{\text { del censo }}{1960} \frac{1}{\text { col } 5}$} & \multicolumn{2}{|c|}{$\begin{array}{l}\text { Indicidores do } \\
\text { seleotjuidad }\end{array}$} \\
\hline & & & & & & $\begin{array}{c}\text { TCol. } 1-4 \\
\text { col. } 6\end{array}$ & $\begin{array}{c}\left(\mathrm{Col}_{0} 3-5\right) \\
\mathrm{Col}_{2} 7 \\
\end{array}$ \\
\hline$\underset{\mathbb{N}}{\text { Categoria I (superior) }}$ & $\begin{array}{l}39 \\
(31)\end{array}$ & $\left(\begin{array}{l}51 \\
35\end{array}\right)$ & $\begin{array}{c}62 \\
(37)\end{array}$ & 48 & 51 & -9 & +11 \\
\hline$\underset{N}{\text { Categoría II }}$ & $\begin{array}{l}25 \\
(24)\end{array}$ & $\begin{array}{l}45 \\
(40)\end{array}$ & $\begin{array}{l}48 \\
(48)\end{array}$ & 14 & 23 & +11 & $\$ 25$ \\
\hline $\begin{array}{l}\text { Categoria III } \\
\text { N }\end{array}$ & $\begin{array}{l}50 \\
(30)\end{array}$ & $\begin{array}{l}31 \\
(35)\end{array}$ & $\begin{array}{l}25 \\
(53)\end{array}$ & 8 & 15 & $\$ 42$ & +10 \\
\hline Categoría IV & $\left(\begin{array}{l}51 \\
(67)\end{array}\right)$ & $\begin{array}{l}32 \\
(76)\end{array}$ & $\begin{array}{l}27 \\
(73)\end{array}$ & 5 & 8 & +46 & +19 \\
\hline Categoría V (inferior) & $\left(\begin{array}{l}34 \\
12)\end{array}\right.$ & $\begin{array}{l}10 \\
(21)\end{array}$ & $\begin{array}{l}13 \\
(31)\end{array}$ & 3 & 6 & +31 & +7 \\
\hline Todas las categorías & $\begin{array}{c}43 \\
(164)\end{array}$ & $\begin{array}{c}35 \\
(207)\end{array}$ & $\begin{array}{c}34 \\
(242)\end{array}$ & 26 & $35^{2 n d x}$ & +17 & -1 \\
\hline Total nacional & & & & 12 & 21 & & \\
\hline
\end{tabular}

* El ordenamiento socioeconómico se formó con base en índices de desarrollo económico (ver texto). Se incluyen en las tabulaciones todas las zonas que contribuyeron con el $2 \%$ o más de los migrantes a Monterrey; juntas dan cuenta del $82 \%$ del total. Las zonas son. Categoria I (Distrito Federal, Piedras Negras-Acuna, Sabinas, Saltillo); Categoria II (Matamoros, Comarca Lagunera y el municipio de Aguascalientes); Categoría III (Coahuila Sur, Montemorelos, Tamaulipas centro); Categoría TV (Bajío, Nucvo León norte, San Luis Potosí norte), Categoría V (Zacatecas excepto la capital, Nuevo león sur).

* Esta cifra es tan elevada porque es una media no ponderada y por lo tanto la categoría I (que contiene a la ciudad de México) contribuye en forma desproporcionada. 
listribución total el grado de selectividad positiva de los migrantes ha disminuido. Se podría decir que estas diferencias entre cohortes. de llegada están muy afectadas por su variación en las distribuciones por edades, como ya se mencionó. Ninguno de los individuos de la cohorte anterior a 1941 pudo haber tenido más de 40 años cuando llegaron a Monterrey, y en esa fecha, como ahora, los individuos más. jóvenes tienen un nivel educativo más alto que los mayores. Sin embargo, cuando se elaboró un cuadro similar sólo para el caso de los migrantes que llegaron antes de los 40 años de edad, los resultados mostraron esencialmente el mismo patrón de selectividad decreciente.

De nuevo el promedio oculta el hecho de que la declinación en la selectividad sólo es cierta para las categorías III a V. Las primeras dos muestran aumento en la selectividad, aunque más moderado que la disminución en las últimas tres categorías. Al evaluar estos resultados, debe tenerse presente que para todo el país durante el período 1940-1960 ocurrieron mejoras sustanciales en el grado de educación, pues el porciento de individuos de más de 15 años de edad con seis o más de escuela se elevó de 12 a 21 . ¿Hay alguna manera de tomar en cuenta las tendencias divergentes representadas en nuestra información?

La respuesta reside en el carácter distinto de las zonas que componen las categorías I y II cuando se comparan con las otras tres. Las primeras dos, como se recordará, son predominantemente urbanas, y la categoría I es metropolitana. Los migrantes de esas zonas a Monterrey rara vez son impelidos a abandonarlas por falta de oportunidades económicas, ya que todas ellas se encuentran entre las de mayor crecimiento y dinamismo en el país. Gran cantidad de los que emigran a Monterrey, provenientes de estas áreas, lo hacen probablemente con la esperanza de mejorar en sus carreras ocupacionales; salvo en el caso excepcional ya aludido, los migrantes son selectivos positivamente. No se sabe por qué las cohortes de llegada de categoría I son excepción, salvo que posiblemente Monterrey, antes de iniciarse el auge industrial de los cuarentas, no era particularmente atractivo para los migrantes que tenían en mente hacer carrera, en comparación con áreas tales como la ciudad de México.

La situación que afrontan los migrantes provenientes de zonas pertenecientes a las categorías III, IV y V es muy distinta. En este caso, los migrantes de fechas tempranas eran de lo que podría llamarse naturaleza pionera, porque eran altamente selectivos respecto de la población de donde provenían; probablemente, aunque nuestra información no nos da apoyo directo sobre este punto, al ser los más educados se percataron mejor de las perspectivas económicas locales poco prometedoras y fueron, por lo tanto, los más deseosos de abandonar el área. Esto no es más que otra ilustración del hecho de que la educación es a menudo estímulo a la emigración. No sólo amplía los horizontes de los que la reciben y los hace conocer otras oportunidades, sino que en general provoca insatisfacción con las oportunidades y condiciones locales. En contraste, las llegadas más recientes a Monterrey representan una migración "en masa" que no difiere sustancialmente de las características de la población de las comunidades 
Cuadro 3

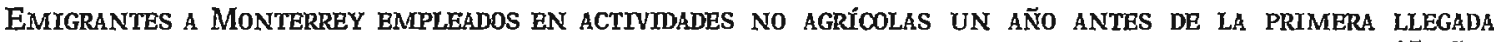
AGRUPADOS POR ZONAS DE COMUNIDAD DE ORIGEN, COMPARADOS CON TODOS LOS INDIVIDUOS EMPLEADOS, DE 15 AÑOS Y MÁS, EN LAS MISMAS ZONAS TOMADAS DE LOS CENSOS DE 1940 Y 1960

(En porcientos)

\begin{tabular}{|c|c|c|c|c|c|c|c|}
\hline \multirow[b]{2}{*}{ 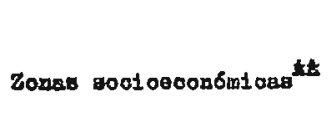 } & \multicolumn{3}{|c|}{ Poriodo de primera $110 \mathrm{gads}$} & \multicolumn{2}{|c|}{ Perrodo dol congo } & \multicolumn{2}{|c|}{$\begin{array}{l}\text { Indlcadores do } \\
\text { Boloctivided }\end{array}$} \\
\hline & $\begin{array}{l}\text { Antes de } 1941 \\
\text { Col. } 1\end{array}$ & $\begin{array}{c}1941-1950 \\
\text { Col.2 }\end{array}$ & $\begin{array}{l}1951-1960 \\
\text { col. } 3\end{array}$ & $\begin{array}{l}1940 \\
\text { Col. } 4\end{array}$ & $\begin{array}{l}1960 \\
\text { Col. } 5\end{array}$ & Col. $\left.{ }^{1-4}\right)$ & $\begin{array}{l}\text { Col. 3-5) } \\
\text { Coi.7 }\end{array}$ \\
\hline Gategoria I (Stporiox) & 76 & 74 & 82 & 89 & 95 & -13 & -13 \\
\hline CatogorIa II & (21) & (23) & $\begin{array}{l}(33) \\
62\end{array}$ & 36 & 41 & +38 & +21 \\
\hline$\stackrel{\text { Ḣ }}{\text { Catogorla III }}$ & (23) & (28) & (39) & 19 & 15 & +49 & +29 \\
\hline II & (19) & $(28)$ & $(45)$ & 20 & 18 & +41 & +24 \\
\hline Categorla IV & (31) & $\left(\begin{array}{l}46 \\
(63)\end{array}\right.$ & $(59)$ & 20 & 10 & & \\
\hline Categorie V & $t$ & $\begin{array}{l}68 \\
(19)\end{array}$ & $\begin{array}{l}24 \\
(29)\end{array}$ & 15 & 13 & - & +11 \\
\hline Todas las oategorfias & $\begin{array}{c}71 \\
(101)\end{array}$ & $\begin{array}{c}56 \\
(161)\end{array}$ & $\begin{array}{r}50 \\
(205)\end{array}$ & 51 & $64^{4 x}$ & +20 & -14 \\
\hline Totel neotonal & & & & 31 & 41 & & \\
\hline
\end{tabular}

* Menos de diez casos.

*t Ver cuadro 2. 
de origen. De aquí el marcado descenso de la magnitud de las diferencias, en puntos de porciento, que aparece en las columnas 6 y 7 .

Hay otro aspecto del cuadro 2 que vale la pena comentar; al examinar las columnas 1-3 el lector notará que en las llegadas más tempranas (columna 1) no hay progresión descendente de las categorías más altas a las más bajas. En contraste, las columnas 2 y 3 muestran un descenso regular y pronunciado. En otras palabras, los migrantes a Monterrey reflejan en forma creciente la posición socioeconómica de las zonas de origen.

En el cuadro 3, donde la variable es el porciento de individuos empleados en actividades no agrícolas, el patrón es similar pero no tan notable como el de la educación; todas las categorías. excepto la I

\section{Cuadro 4}

CARACTERfSTTCAS ESCOGIDAS DE EMIGRANTES ENTRE 20 Y 29 Y 30 Y 39 AÑNS DE EDAD AI. LLEGAR POR PRTMERA VEZ A MONTERREY, AGRUPADOS POR PERIODOS DE PRIMERA LIEGADA

(En porcientos)

\begin{tabular}{|c|c|c|c|}
\hline \multirow{2}{*}{$\begin{array}{l}\text { Caraoterfałions un } \\
\text { año antes do le } \\
\text { llegada }\end{array}$} & \multicolumn{3}{|c|}{ Pridars Llegeda } \\
\hline & Antes de 1941 & 1941-1959 & $1951-1960$ \\
\hline Patson, por au ougnto & (73) & $\begin{array}{l}(13 \\
(87)\end{array}$ & $\begin{array}{l}11 \\
(80)\end{array}$ \\
\hline Edad: $\quad{ }_{\mathrm{R}}^{30-39}$ & (13) & $(43)$ & $\begin{array}{c}13 \\
(71)\end{array}$ \\
\hline $\begin{array}{l}\text { Bugraoion Dtroota a } \\
\text { Monterrog }\end{array}$ & & & \\
\hline $\begin{array}{c}20-29 \\
y \\
\text { Pads: } \\
30-39 \\
y\end{array}$ & $\begin{array}{l}40 \\
(73) \\
23 \\
(13)\end{array}$ & $\begin{array}{r}58 \\
(87) \\
40 \\
(48)\end{array}$ & $\begin{array}{l}50 \\
(80) \\
38 \\
(71)\end{array}$ \\
\hline $\begin{array}{c}\text { Soltarod } \\
20-29 \\
x\end{array}$ & $\begin{array}{c}66 \\
(73)\end{array}$ & $\begin{array}{l}57 \\
(87)\end{array}$ & $\begin{array}{l}65 \\
(80)\end{array}$ \\
\hline Bdad $\quad \frac{30-39}{X}$ & (13) & $\begin{array}{c}19 \\
(48)\end{array}$ & $\left(\begin{array}{c}13 \\
(71)\end{array}\right.$ \\
\hline 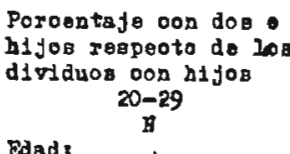 & (13) & $(27)$ & $\begin{array}{c}39 \\
(18)\end{array}$ \\
\hline$\stackrel{30-39}{\times}$ & 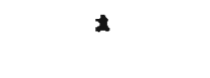 & $\begin{array}{c}81 \\
(32)\end{array}$ & $\begin{array}{l}86 \\
(58)\end{array}$ \\
\hline 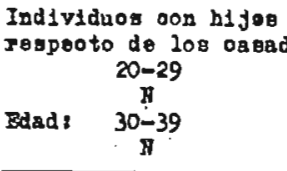 & $\begin{array}{l}52 \\
(25) \\
50 \\
(10)\end{array}$ & $\begin{array}{l}73 \\
(37) \\
84 \\
(38)\end{array}$ & $\begin{array}{l}64 \\
(28) \\
93 \\
(69)\end{array}$ \\
\hline
\end{tabular}

* Menos de diez casos. 
son selectivas positivamente. La explicación de la selectividad negativa de los migrantes de la categoría I es que mientras que las categorías socioeconómicas están en términos de la comunidad de origen, la clasificación de empleos de los hombres está basada en el lugar donde trabajaban durante el año anterior a la llegada a Monterrey. Parece que algunos hombres se habían mudado de sus áreas metropolitanas y urbanas de origen hacia otras comunidades en donde llevaron a cabo trabajos agrícolas. Mientras que la disminución en la selectividad de los migrantes entre 1940 y 1960 no aparece tan acentuada en este cuadro como la correspondiente a educación en el cuadro 2, el punto más importante es que Monterrey atrae en forma creciente a aquellos que tienen directamente antecedentes agrícolas, esto a pesar del hecho de que tanto para todo el país como para el nordeste de México en particular, el empleo agrícola ha declinado relativamente durante este período de 20 años.

\section{Características Seleccionadas de las COHORTES}

Al comparar los datos del censo con los de la encuesta de Monterrey hemos demostrado que los migrantes son en verdad selectivos respecto de las poblaciones de donde provienen. ¿Pero cómo arrojar más luz sobre la cuestión de las diferencias entre los migrantes "en masa" y los "pioneros"? Los datos censales son ayuda limitada así que debemos acudir a información proveniente sólo de la encuesta de Monterrey. Todas las cinco variables presentadas en el cuadro 4 están influidas por la edad, y por ello controlamos este factor considerando sólo individuos entre 20 y 29 y 30 y 39 años de edad en la fecha de llegada. Los grupos de edad más jóvenes y más viejos tenían tan pocos casos que no valió la pena considerarlos.

El autoempleo en las actividades no agrícolas se seleccionó porque puede anticiparse la hipótesis de que los migrantes "pioneros" de fechas más tempranas estaban sobrerrepresentados por pequeños comerciantes y dependientes de tiendas de áreas rurales o de centros urbanos pequeños que serían probablemente los más al tanto de las oportunidades en el exterior y los más ambiciosos por mejorar su situación. El grupo de edad 30-39 da apoyo a esta hipótesis, en virtud de que es dudoso que las dos cohortes de llegada más temprana tengan mucha más representación que la distribución de la fuerza de trabajo en las comunidades de origen. No es evidente el porqué de la casi inexistente variación por fecha de llegada en el grupo de edad 20-29.

La siguiente variable, migración directa a Monterrey, se incluye porque nos permite conocer si haber vivido en otras comunidades distintas de la de origen es parte de los antecedentes de los migrantes. Parece que las cohortes de llegada más reciente, en ambos grupos de edad, con mayor probabilidad constan de individuos que emigraron directamente a Monterrey. Muchos, desde luego, vinieron directamente de áreas rurales.

Las tres variables finales están todas relacionadas con el estado marital. Podemos suponer que aquellos que llegan solteros a Monterrey representan el patrón pionero más que el de masas. Para el 
grupo de edad más joven no hay tendencia aparente en el tiempo. La categoría de edad 30-39, la más vieja, muestra una proporción decreciente de solteros. En cuanto a los individuos casados al llegar, en ambos grupos de edad es cada vez más creíble el que hayan tenido hijos poco después de la llegada aunque en el grupo 20-29 la tendencia es irregular. De aquellos que llegaron con niños, las llegadas más recientes con mayor probabilidad eran personas con dos o más hijos. E1 número de entrevistaclos que llegaron antes de 1941 es tan pequeño que esta categoría debe șer interpretada con precaución. Tomadas juntas, hay consistencia en la conducta de las tres variables, lo cual sugiere que ha habido selectividad decreciente en el tiempo, y esto, por supuesto, es consistente con los resultados previamente presentados. Ciertamente, para todo México, no hay evidencia conocida de que los patrones matrimoniales y familiares mismos hayan experimentado algún cambio durante la generación pasada.

La selectividad de los migrantes en el tiempo puede también observarse en términos del grado educativo de sus padres. En seguida se presenta, para cada cohorte de llegada de los hijos, el porciento de padres y madres que habían completado la primaria:

\begin{tabular}{lccc}
\hline & Antes de 1941 & $1941-50$ & $1951-60$ \\
\hline Padres & 35 & 29 & 26 \\
Madres & 34 & 26 & 24 \\
\hline
\end{tabular}

Es muy claro que el patrón manifestado por los hijos es también característico de sus padres: un grado de educación general por encima del promedio con una pronunciada declinación de las llegadas más tempranas a las más recientes. Si se recuerda que todos los padres fueron educados antes de 1940 y la mayoría antes de 1930, su selectividad es sorprendente, porque es dudoso que más del $8 \%$ de cualquier sexo de la población total mayor de 15 años hubiera completado la primaria antes de 1930. En otras palabras, la selectividad positiva que descubrimos en nuestros entrevistados masculinos también debe estar presente en sus padres.

\section{CONCLUSTONES}

Podemos resumir nuestros hallazgos sobre la selectividad de los emigrantes como sigue: en general, los migrantes son selectivos positivamente respecto de las poblaciones de donde se originan. En esta medida los hallazgos de Blau y Duncan acerca de los Estados Unidos están corroborados. Monterrey ha sido capaz de atraer migrantes de status altos de otras áreas metropolitanas de México, como lo indica el patrón mostrado por la categoría I. El segundo hallazgo en importancia es que los migrantes han perdido selectividad en el transcurso del tiempo, debido en gran parte a la contribución de las zonas más rurales y atrasadas (categorías III-V). Es quizá este patrón el más sorprendente de nuestro estudio y el que amerita una explicación extensa. ¿Cuáles fueron las condiciones que propiciaron la alta selecti- 
vidad en todas las categorías en los períodos más tempranos y qué hay detrás de la emigración "en masa" menos diferencial de las áreas atrasadas en los períodos recientes?

Los emigrantes a Monterrey que llegaron antes de 1941 lo hicieron durante un período de ajuste largo y doloroso a los efectos de la revolución de 1910. Aunque fue poca la violencia entre 1920 y 1940 , durante este tiempo el gobierno central era débil y luchaba por afirmarse frente a caudillos regionales. La economía se había deteriorado a partir de los niveles prerrevolucionarios y la recuperación fue naturalmente obstaculizada por la depresión internacional de los treintas. El verdadero auge económico de México no se inició hasta la segunda guerra mundial. Fueron necesarios casi dos decenios (1921-1940) para que el Monterrey metropolitano duplicara su población, la cual creció de alrededor de 88000 a 186000 y el movimiento migratorio durante este período debió ser una experiencia "pionera" en mayor medida que para las generaciones subsecuentes. Como se sugirió antes, aquellos pocos individuos habitantes de áreas rurales y de pequeños poblados en el nordeste de México que de alguna manera habían obtenido buena educación, indudablemente con mayor probabilidad podían darse cuenta cle las oportunidades en ciudades grandes como Monterrey y podían tener la motivación y el deseo de salir.

Antes de 1941, ir a Monterrey no era ni remotamente tan fácil como lo es ahora. Las carreteras pavimentadas eran escasas y el medio común de transporte a largas distancias, el ferrocarril, tenía rutas limitadas y fue seriamente dañado durante la violencia revolucionaria. Los periódicos y los radiorreceptores eran poco comunes fuera de las ciudades más grandes. En fin, el movimiento migratorio en esta época requirió de consiclerable iniciativa y como tal era selectiva respecto de aquellos mejor preparados y más motivados a mejorar su situación.

En contraste, las llegadas a Monterrey más recientes vieron menos aventura en la migración. El auge que primero sobrevino como consecuencia de la segunda guerra mundial y que ha continuado en gran medida, generó en las ciudades empleos que antes no existían, incluidos empleos en la construcción y en otros trabajos que requerían poca habilidacl. El gran programa de construcción de carreteras y la densa red de rutas de autobuses que trajo consigo, hizo que las jornadas de cientos de lugares a Monterrey fueran más fáciles y también relativamente más baratas. La mayor diseminación de los medios de comunicación masiva, en parte consecuencia de la electrificación, llevó el mundo exterior a las comunidades aisladas y con ello despertó primero el interés y después el deseo de mudarse a las ciudades. Indudablemente, los movimientos de "braceros" que enviaron a Estados Unidos a cientos de miles de mexicanos, muchos de ellos del norte de México, contribuyeron a hacer de los viajes largos algo relativamente común en infinidad de poblados mexicanos.

Los factores mencionados ayudan a explicar la selectividad decreciente. Pero hay otro factor no mencionado hasta ahora que es muy importante para mostrar por qué la selectividad debe casi inevitablemente disminuir. Considérese el hecho de que la población de Monterrey aumentó de 134000 en 1930 a 950000 habitantes (estimación) 
en 1965. El aumento intercensal entre 1930 y 1940 fue de 54000 ; entre 1940 y 1950 de 170000 y de 1950 a 1960 de 324000 . Suponiendo, sólo para los propósitos de nuestro argumento, que la migración neta contribuyó con la mitad de los aumentos de cada década, el número de emigrantes entre la primera y última décadas se sextuplicó, de 27000 a 162000 . Esto significa que las demandas puestas sobre las "reservas" de emigrantes potenciales habian estado aumentando en forma sostenida (Monterrey no ha monopolizado el acceso a dicha reserva, ya que muchas otras ciudades del nordeste de México, especialmente las que están cerca de o en la frontera, han tenido crecimiento muy rápido). Ahora bien, muchos de los que emigraron a Monterrey son de origen rural; el $56 \%$ era de lugares con menos de 5000 habitantes. No obstante, dicha reserva ha crecido lentamente desde 1930. Si tomamos los tres estados del nordeste de México (Nuevo León, Coahuila y San Luis Potosí), que representaron el lugar de nacimiento del $74 \%$ de todos los migrantes, la población rural total (definida como la de localidades menores de 5000 ) fue de 956000 en 1930, 1169000 en 19401343000 en 1950 y 1463000 en 1960 . Este aumento del $53 \%$ en 30 años es mucho menor que las "demandas" generadas por la tasa de crecimiento de Monterrey. Por lo tanto, es de esperar una "regresión hacia la media". Todas las partes de la "reserva" de población se ven afectadas en forma creciente, no sólo la población soltera con mejor educación.

Aún está en pie la pregunta sobre si el patrón de selectividad de Monterrey se presenta en otros centros urbanos en México o en otros países latinoamericanos. Ciertamente la tasa de crecimiento económico de la ciudad tendrá su efecto, y el crecimiento de Monterrey durante las últimas tres décadas es difícil de igualar. En comunidades estancadas económicamente el patrón de selectividad puede ser diferente, porque los migrantes de status más alto pasarán por alto estos lugares en su camino hacia las ciudades de mayor promisión. Por desgracia, se sabe muy poco acerca de los patrones migratorios de esta clase de ciudades aunque sabemos, sin embargo, que muchas ciudades de América Latina acusan crecimiento de población acelerado aun cuando sus economías no son robustas. Esto está vinculado a los altos niveles de crecimiento natural de las zonas de influencia - principalmente rurales- de esas ciudades que crea una presión ascendente sobre los recursos locales, lo cual a su vez estimula la emigración hacia las ciudades.

Nosotros, por lo tanto, sostenemos que el patrón de selectividad de Monterrey no es único, sino que será encontrado en otras áreas grandes y de crecimiento elevado tanto en México como en otros países de América Latina. En particular, las oportunidades relativamente más prometedoras y las presiones de la población sobre los recursos rurales, combinadas con el número cada vez mayor de emigrantes necesario para sustentar el crecimiento elevado de las grandes ciudades llevará al patrón de selectividad decreciente que hemos encontrado en Monterrey.

Si esta predicción resulta cierta deberá tener implicaciones muy importantes en el curso del desarrollo de las ciudades latinoamericanas. Sugiere, en efecto, que la asimilación de los migrantes a los gran- 
des núcleos urbanos de América Latina se tornará más difícil. Por una parte, el aumento de los migrantes menos selectivos traerá consigo menos educación y calificación, y tal vez menos motivación para adaptarse al ambiente urbano. Por otra parte, las elevadas tasas de crecimiento natural de las ciudades elevan la proporción de nativos y probablemente la competencia por mejores empleos entre aquéllos y los migrantes aumentará en intensidad. Por añadidura, hay evidencia de que en los empleos mejores se requiere cada vez más de certifícados de estudios formales tales como la primaria y aun la secundaria, inclusive en los empleos manuales calificados.7 Esto pone en seria desventaja a los migrantes de áreas rurales donde las facilidades educativas faltan o son muy pobres. Lo que resulta probable es, por lo tanto, que no obstante que los migrantes serán una proporción progresivamente menor de la población de las ciudades latinoamericanas, el problema de la asimilación de esas personas' a la estructura social y ocupacional de las ciudades será cada vez más grave.

Traducción de A. García Rocha

7 Jorge Balán, "Migrant-native socio-economic differences in Latin American cities: a structural analysis", por aparecer en Latin American Research Review, Austin, Texas. 